\title{
SECRET CORRELATION IN REPEATED GAMES WITH IMPERFECT MONITORING
}

\author{
OLIVIER GOSSNER AND TRISTAN TOMALA
}

\begin{abstract}
We characterize the maximum payoff that a team can guarantee against another in a class of repeated games with imperfect monitoring. Our result relies on the optimal trade-off for the between optimization of stagepayoffs and generation of signals for future correlation.
\end{abstract}

\section{INTRODUCTION}

It many strategic situations, a group of players may find it beneficial to coordinate their action plans in a way that is hidden from other players. The manager of a sport team devices coordinated plans for the team members, and a choice between them is made during the course of the game. Generals of allied armies need to keep their coordinated plans secret from ennemies. On the internet, coordinated attacks of systems (e.g. by viruses) are known to be much more dangerous than uncoordinated ones. The management of a firm coordinates the actions of the units in a way that is hidden from the competition.

Coordination of a group of players needs to rely on the observation of a common signal by its members. This signal can arise from an external correlation device (Aumann, [Aum74]), or be the result of communication between the players (Forges, [For86]). In a repeated game with imperfect monitoring, players observe random and correlated signals (deterministic signals is a particular case) that depend on chosen actions. These games therefore represent both correlated signals and communication possibilities.

This article explores the possibilities of secret correlation between team members in a repeated game with imperfect monitoring. Our model opposes a team of players called team $I$ to another team called team $I I$ viewed as a single player. Team I's member's action sets are denoted $A^{i}, i \in I$, and team $I I$ 's action set is $B$. At each stage, team $I I$ observes a (possibly random) signal $s$ about $I$ 's actions $a$ drawn according to some probability distribution $q(s \mid a)$. Team $I$ 's members are informed of $a, s$, and possibly of $I I$ 's actions (our result covers the cases in which team $I$ has perfect, imperfect, or no observation of $I I$ 's choice). The payoff to team $I$ is a function of both team's action choices. In order to stress the value of secret correlation between team members, we assume that team II's goal is to minimize team $I$ 's payoff. Since team $I$ has more information than team $I I$ about action choices, this extra information can be used as a correlation device for future actions. Our model allows to study the optimal trade-offs for team $I$ between generation of signals for future correlation and use of correlation for present payoffs.

Our main result is a characterization of the best payoff that the team can guarantee against outside players (both for limits of discounted payoffs, or limits of mean payoffs, and for uniform payoffs). We emphasize three reasons why characterizing the max min value is important.

Date: 10th March 2004 
First, the max min of the repeated game measures how successful team $I$ is in correlating secretly its actions from outside players. Indeed, when no correlation is possible, the max min of the repeated game coincides with the max min in mixed strategies of the stage game. When full correlation is achievable, this max min equals the generally higher max min in correlated strategies of the stage game. In general, partial correlation only may be achievable, and the max min of the repeated game may lie between these two values.

Second, von Stengel and Koller [vSK97] proved that, in finite games opposing a team of players to one outside player, the max min payoff is a Nash payoff. Furthermore, it is the most natural Nash payoff to select since team members can guarantee this value. Combined with our result, we know that the maximal Nash payoff to the team in the $n$-stage games with imperfect monitoring is the max min we characterize.

Finally, characterization of max min payoffs in repeated games, are important for the general study of repeated games with imperfect monitoring. Indeed, the message of the "Folk Theorem" is that in repeated games with perfect monitoring and sufficiently little discounting, the set of equilibrium payoffs is given by the set of feasible and individually rational payoffs of the one-shot game. Such payoffs can be enforced by a plan in which players follow a path generating the desired payoff, and any player that deviates from this plan gets punished by the others to his individually rational level. Generalizations of the "Folk Theorem" to games with imperfect monitoring yield two types of questions. First, the signalling structure may render deviations deviations undetectable, so one needs to characterize detectable deviations. And second, assuming it is commonly known that a player $i$ has deviated, how harsh can this player be punished? This last question amounts to characterizing the min max of player $i$ in the repeated game, or the max min of the team of players trying to minimize $i$ 's payoff.

The problem faced by the team consists in finding the optimal tradeoff between using previous signals that are unknown to team $I I$ as correlation devices, and generating such signals for future use. We measure the amount of secret information contained of past signals by their entropy. Our main results characterizes the team's max min payoff as the best payoff that can be obtained as a convex combination of correlated strategies under the constraint that the average entropy spend in correlation devices does not exceed the average entropy of secret signals generated.

We motivate the problem by discussing examples in section 2, present the model and tools in section 3, and the main result in section 4 . We illustrate our result in the simple cases of perfect and trivial observations in section 5, discuss computational applications in section 6 , show an example of a signalling structure for which a folk theorem obtains in section 7 , and conclude with extensions in section 8 .

\section{EXAMPLES}

We consider a 3 -player game where the team is $I=\{1,2\}$ opposing player $I I=\{3\}$. Player 1 chooses rows, player 2 chooses columns and player 3 chooses matrices. Consider the following one-shot game:

$$
\begin{aligned}
& a \\
& b \\
& L
\end{aligned} \frac{\left.\begin{array}{ll}
a & b \\
1 & 0 \\
0 & 0
\end{array}\right)}{R}
$$

In the repeated game with perfect monitoring, the team guarantees the max min of the one-shot game, where the max runs over the independent probability distributions on $A^{1} \times A^{2}$ that is the team guarantess $\frac{1}{4}$. 
Assume now that player 3 recieves blank signals, i.e. has no information on the action profile of $I$, whereas players 1 and 2 observe each other's actions. The team can then use the first move of player 1 as a correlation device and thus guarantee the max min of the one-shot game, where the max runs over the set of all probability distributions on $A^{1} \times A^{2}$ that is from the second stage on, $I$ guarantees $\frac{1}{2}$.

Consider now the case where team members observe each other's actions and the signal of player 3 is given by the following matrix:

$$
\begin{aligned}
& a \\
& b
\end{aligned} \quad\left(\begin{array}{cc}
a & b \\
s & s^{\prime} \\
s^{\prime} & s
\end{array}\right)
$$

Player 3 thus learns at each stage whether player 1 and 2 played the same action. Consider the following strategy of the team: play the mixed action profile $\left(\frac{1}{2}, \frac{1}{2}\right) \otimes\left(\frac{1}{2}, \frac{1}{2}\right)$ at the first stage and let $\mathbf{a}_{1}^{1}$ be the random move of player 1 at stage 1 . At each stage $n>1$, play $(a, a)$ if $\mathbf{a}_{1}^{1}=a$ and play $(b, b)$ if $\mathbf{a}_{1}^{1}=a$. The signal of player 3 at stage 1 is distributed $\left(\frac{1}{2}, \frac{1}{2}\right)$ and conditional on this signal, $\mathbf{a}_{1}^{1}$ is also distributed $\left(\frac{1}{2}, \frac{1}{2}\right)$. Since after stage 1 the signals will be constant, player 3 never learns anything about the value of $\mathbf{a}_{1}^{1}$. Players 1 and 2 are thus correlated from stage 2 on and $I$ guarantees $\frac{1}{2}$.

Finally consider now the case where team members observe each other's actions and the signal of player 3 is given by player 2's action, i.e. by the following matrix:

$$
\begin{aligned}
& a \\
& b
\end{aligned}\left(\begin{array}{cc}
a & b \\
s & s^{\prime} \\
s & s^{\prime}
\end{array}\right)
$$

As is the previous case, the move $\mathbf{a}_{1}^{1}$ of player 1 at stage 1 is unobserved by player 3 and may serve as a correlation device. Again let the team play the mixed action profile $\left(\frac{1}{2}, \frac{1}{2}\right) \otimes\left(\frac{1}{2}, \frac{1}{2}\right)$ at the first stage and at stage 2 , play $(a, a)$ if $\mathbf{a}_{1}^{1}=a$ and $(b, b)$ if $\mathbf{a}_{1}^{1}=a$. However, the move of player 2 at stage 2 reveals $\mathbf{a}_{1}^{1}$ and thus the correlation is lost after stage 2 . The trade-off between generating signals for correlation and using this correlation appears here, the first stage generates a correlation device and the second uses it. Playing this two-stage strategy cyclically, the team guarantees $\frac{3}{8}$ and we shall see that this is not optimal.

\section{MODEL AND DEFINITIONS}

3.1. The repeated game. Let $I=\{1, \ldots,|I|\}$ be a finite set of players called team and $I I$ be another player. For each player $i \in I$, let $A^{i}$ be player $i$ 's finite set of actions and let $B$ be player $I I$ 's finite set of actions. We denote $A=\prod_{i \in I} A^{i}$. At each stage $t=1,2, \ldots$, each player chooses an action in his own set of actions and if $(a, b)=\left(\left(a^{i}\right)_{i \in I}, b\right) \in A \times B$ is the action profile played, the payoff for each team player $i \in I$ is $g(a, b)$ where $g: A \times B \rightarrow \mathbb{R}$. The payoff for player $I I$ is $-g(a, b)$.

After each stage, if $a$ is the action profile played by players $i \in I$, a signal $s$ is drawn in a finite set $S$ according to $q(s \mid a)$, where $q: A \rightarrow \Delta(S)$. Player $I I$ observes $(s, b)$, whereas team players observe $(a, s, b)$. Thus, in our model all team members observe the same random signal that reveals the signal observed by player $I I$.

A history of length $n$ for the team is an element $h_{n}$ of $H_{n}=(A \times B \times S)^{n}$, and a history of length $n$ player $I I$ is $h_{n}^{I I}$ element of $H_{n}^{I I}=(B \times S)^{n}$. For each finite set $X$, we let $\Delta(X)$ be the set of probabilities on $X$. A behavioral strategy $\sigma^{i}$ for a team player $i$ is a mapping $\sigma^{i}: \cup_{n} H_{n} \rightarrow \Delta\left(A^{i}\right)$ and a behavioral strategy $\tau$ for player $I I$ is a mapping $\tau: \cup_{n} H_{n}^{I I} \rightarrow \Delta(B)$. A profile of behavioral strategies $(\sigma, \tau)=\left(\left(\sigma^{i}\right)_{i \in I}, \tau\right)$ induces a probability distribution $\mathbf{P}_{\sigma, \tau}$ on the set of plays $(A \times B \times S)^{\infty}$ endowed with the product $\sigma$-algebra. 
Given a discount factor $0<\lambda<1$, the discounted payoff for team $I$ induced by $(\sigma, \tau)$ is: $\gamma_{\lambda}(\sigma, \tau)=\mathbf{E}_{\sigma, \tau}\left[\sum_{n}(1-\lambda) \lambda^{n-1} g\left(\mathbf{a}_{n}, \mathbf{b}_{n}\right)\right]$ where $\left(\mathbf{a}_{n}, \mathbf{b}_{n}\right)$ denotes the random action profile at stage $n$. The $\lambda$-discounted max min payoff of team $I$ denoted $v_{\lambda}$ is:

$$
v_{\lambda}=\max _{\sigma} \min _{\tau} \gamma_{\lambda}(\sigma, \tau)
$$

The average payoff for team $I$ up to stage $n$ is: $\gamma_{n}(\sigma, \tau)=$ $\mathbf{E}_{\sigma, \tau}\left[\frac{1}{n} \sum_{m=1}^{n} g\left(\mathbf{a}_{n}, \mathbf{b}_{n}\right)\right]$. The $n$-stage max min payoff of team $I$ denoted $v_{n}$ is:

$$
v_{n}=\max _{\sigma} \min _{\tau} \gamma_{n}(\sigma, \tau)
$$

The max min payoff of player $I I$ in the non-discounted infinitely repeated game, denoted $v_{\infty}$ is defined in the usual way (see Mertens Sorin Zamir [MSZ94]):

(1) The team $I$ guarantees $v \in \mathbb{R}$ if:

$$
\forall \varepsilon>0, \exists \sigma=\left(\sigma^{i}\right)_{i \in I}, \exists N \text { s.t. } \forall \tau, \forall n \geq N, \gamma_{n}(\sigma, \tau) \geq v-\varepsilon .
$$

(2) Player $I I$ defends $v \in \mathbb{R}$ if: $\forall \varepsilon>0, \forall \sigma, \exists \tau, \exists N$ s.t. $\forall n \geq N, \gamma_{n}(\sigma, \tau) \leq v+\varepsilon$.

(3) The uniform max min, if it exists, is $v_{\infty} \in \mathbb{R}$ such that $I$ guarantees $v_{\infty}$ and $I I$ defends $v_{\infty}$.

3.2. Best replies and Autonomous strategies. We define first stratgies for player $I I$ that play myopic best replies.

Definition 1. Let $\sigma$ be a strategy for the team, define inductively $\tau_{\sigma}$ as the strategy of player II that plays stage-best replies to $\sigma$ :

At stage $1, \tau_{\sigma}(\emptyset) \in \operatorname{argmin}_{b} g(\sigma(\emptyset), b)$ where $\emptyset$ is the null history that starts the game. Assume that $\tau_{\sigma}$ is defined on histories of length less that $n+1$. For every history $h_{n}^{I I}$ of player II, let $x_{n+1}\left(h_{n}^{I I}\right) \in \Delta(A)$ be the distribution of the action profile of the team at stage $n+1$ given $h_{n}^{I I}$ and let $\tau_{\sigma}\left(h_{n}^{I I}\right)$ be in $\operatorname{argmin}_{b} g\left(x_{n+1}\left(h_{n}^{I I}\right), b\right)$.

We introduce now a class of strategies for the team against which the myopic best reply is a best reply in the repeated game. Call a strategy of a team player autonomous if it does not depend on player $I I$ 's past moves that is for $i \in I$, $\sigma^{i}: \bigcup_{n}(A \times S)^{n} \rightarrow \Delta\left(A^{i}\right)$. Against a profile of autonomous strategies, the myopic best reply is a true best reply.

Lemma 2. Let $\sigma$ be a profile of autonomous strategies, for each stage $n$ and strategy $\tau$ for player II, $\mathbf{E}_{\sigma, \tau_{\sigma}} g\left(\mathbf{a}_{n}, \mathbf{b}_{n}\right) \leq \mathbf{E}_{\sigma, \tau} g\left(\mathbf{a}_{n}, \mathbf{b}_{n}\right)$ and thus $\tau_{\sigma}$ is player II's best reply in any version of the repeated game.

Proof. Consider the optimization problem of player $I I$,

$$
\min _{\tau} \sum_{n=1}^{N} \mathbf{E}_{\sigma, \tau} g\left(\mathbf{a}_{n}, \mathbf{b}_{n}\right)
$$

Since player $I I$ 's moves do not influence the play of the team, this amounts to solve for each $n$ and history $h_{n}^{I I}, \min _{b} \mathbf{E}_{\sigma}\left[g\left(\mathbf{a}_{n}, b\right) \mid h_{n}^{I I}\right]$. The same argument applies in the discounted game.

3.3. Information theory tools. The entropy of $\mathbf{x}$ finite random variable with law $P$ is by definition:

$$
H(\mathbf{x})=-\mathbf{E}[\log P(\mathbf{x})]=-\sum_{x} P(x) \log P(x)
$$

where log denotes the logarithm with base 2 . Note that $H(\mathbf{x}) \geq 0$ and that $H(\mathbf{x})$ depends only on the law $P$ of $\mathbf{x}$. The entropy of $\mathbf{x}$ is thus the entropy $H(P)$ of its distribution $P$, with $H(P)=-\sum_{x} P(x) \log P(x)$. 
Let $(\mathbf{x}, \mathbf{y})$ be a couple of random variables with joint law $P$ such that $\mathbf{x}$ is finite. The conditional entropy of $\mathbf{x}$ given $\{\mathbf{y}=y\}$ is the entropy of the conditional distribution $P(\mathbf{x} \mid y)$ :

$$
H(\mathbf{x} \mid y)=-\mathbf{E}[\log P(\mathbf{x} \mid y)]
$$

The conditional entropy of $\mathbf{x}$ given $\mathbf{y}$ is the expected value of the previous:

$$
H(\mathbf{x} \mid \mathbf{y})=\int H(\mathbf{x} \mid y) d P(y)
$$

If $\mathbf{y}$ is also finite, one has the following relation of additivity of entropies:

$$
H(\mathbf{x}, \mathbf{y})=H(\mathbf{y})+H(\mathbf{x} \mid \mathbf{y})
$$

\section{The Main Result}

The max min values $v_{\lambda}, v_{n}, v_{\infty}$ are defined in terms of the data of the repeated game. Our main result is a characterization of these for sufficiently long games in terms of the data of the one-stage game.

4.1. Correlations systems. Let $\sigma$ be a strategy. Suppose that at stage $n$, the history for player $I I$ is $h_{n}^{I I}=\left(b_{1}, s_{1}, \ldots, b_{n}, s_{n}\right)$. Let $h_{n}=\left(a_{1}, b_{1}, s_{1}, \ldots, a_{n}, b_{n}, s_{n}\right)$ be the history for the team. The mixed action played by the team at stage $n+1$ is $\sigma\left(h_{n}\right)=\left(\sigma^{i}\left(h_{n}\right)\right)_{i \in I}$. We denote by $x$ this vector which is unknown to player $I I$ who holds a belief on the value of $x$. Namely, $x=\sigma\left(h_{n}\right)$ with probability $\mathbf{P}_{\sigma}\left(h_{n} \mid h_{n}^{I I}\right)$. The distribution of the action profile $\mathbf{a}_{n+1}$ given the information $h_{n}^{I I}$ of player $I I$ is $\bar{x}=\sum_{h_{n}} \mathbf{P}_{\sigma}\left(h_{n} \mid h_{n}^{I I}\right) \sigma\left(h_{n}\right)$ the barycenter of $x$, element of $\Delta(A)$ the set of correlated distribution on $A$.

Definition 3. Let $X=\otimes_{i \in I} \Delta\left(A^{i}\right)$ be the set of independent probability distributions on $A$. A correlation system is a probability distribution on $X$ and we let $C=\Delta(X)$ be the set of all correlation systems.

$X$ is a closed subset of $\Delta(A)$ and thus $C$ is compact with respect to the weak-* topology.

Assume that at some stage $n$, after some history $h_{n}^{I I}$, the distribution of $\sigma\left(h_{n}\right)$ given $h_{n}^{I I}$ is $c$. The play of the game at this stage is as if: $h_{n}$ were drawn with probability distribution $c$ and announced to each player of the team but not to player $I I$ and, given $h_{n}$, each team player chooses a mixed action. This generates a random action profile for the team and a random signal. We study the variation of uncertainty of player II regarding the total history, measuring uncertainty by entropy.

Definition 4. Let $c$ be a correlation system and $(\mathbf{x}, \mathbf{a}, \mathbf{s})$ be a random variable in $X \times A \times S$ such that the law of $\mathbf{x}$ is $c$, the law of $\mathbf{a}$ given $\{\mathbf{x}=x\}$ is $x$ and the law of $\mathbf{s}$ given $\{\mathbf{a}=a\}$ is $q(\cdot \mid a)$. The entropy variation of $c$ is:

$$
\Delta H(c)=H(\mathbf{a}, \mathbf{s} \mid \mathbf{x})-H(\mathbf{s})
$$

The entropy variation is the difference between the entropy gained by the team and the entropy lost. The entropy gain is the additional uncertainty contained in $(\mathbf{a}, \mathbf{s})$; the entropy loss is the entropy of $\mathbf{s}$ which is observed by player $I I$. If $\mathbf{x}$ is finite, from the additivity formula:

$$
H(\mathbf{x}, \mathbf{a}, \mathbf{s})=H(\mathbf{x})+H(\mathbf{a}, \mathbf{s} \mid \mathbf{x})=H(\mathbf{s})+H(\mathbf{x}, \mathbf{a} \mid \mathbf{s})
$$

and therefore,

$$
\Delta H(c)=H(\mathbf{x}, \mathbf{a} \mid \mathbf{s})-H(\mathbf{x})
$$

The entropy variation is thus the new entropy minus the initial entropy. 
We define now, given a correlation system $c$, the payoff obtained when player $I I$ plays a best reply to the expected distribution on $A$.

Definition 5. Given a correlation system $c$, the distribution of the action profile for the team is $x_{c}=\int_{X} x d c(x) \in \Delta(A)$. The optimal payoff yielded by $c$ is $\pi(c)=\min _{b \in B} g\left(x_{c}, b\right)$, where $g$ is extended to mixed actions in the usual way.

We consider the set of feasible vectors $(\Delta H(c), \pi(c))$ in the (entropy variation, payoff) plane:

$$
V=\{(\Delta H(c), \pi(c)) \mid c \in C\}
$$

Lemma 6. $V$ is compact.

Proof. Since the signal s depends on a only, the entropy variation is:

$$
\Delta H(c)=H(\mathbf{a} \mid \mathbf{x})+H(\mathbf{s} \mid \mathbf{a})-H(\mathbf{s})
$$

From the defintions of entropy and conditional entropy:

$$
\Delta H(c)=\int H(x) d c(x)+\sum_{a} x_{c}(a) H(q(\cdot \mid a))-H\left(\sum_{a} x_{c}(a) q(\cdot \mid a)\right)
$$

which is clearly a continuous function of $c . \Delta H$ and $\pi$ are thus continuous on the compact $C$ and $V$ is compact.

We introduce the following notation:

$$
w=\sup \left\{x_{2} \in \mathbb{R} \mid\left(x_{1}, x_{2}\right) \in \operatorname{co} V, x_{1} \geq 0\right\}
$$

This is the highest payoff associated to a convex combination of correlations systems under the constraint that the average entropy variation is non-negative. For every correlation system $c$ such that $\mathbf{x}$ is a.s. constant, $\Delta H(c) \geq 0$ thus $V$ intersects the half-plane $\left\{x_{1} \geq 0\right\}$ and since $V$ is compact the supremum is indeed a maximum. The number $w$ can also be expressed through the boundary of co $V$. Define for each real number $h$ :

$$
u(h)=\max \{\pi(c) \mid c \in C, \Delta H(c) \geq h\}
$$

From the definition of $V$ we have for each $h$ :

$$
u(h)=\max \left\{x_{2} \mid\left(x_{1}, x_{2}\right) \in V, x_{1} \geq h\right\}
$$

Since $V$ is compact, $u(h)$ is well defined. Let cav $u$ be the least concave function pointwise greater than $u$. Then:

$$
\sup \left\{x_{2} \in \mathbb{R} \mid\left(x_{1}, x_{2}\right) \in \operatorname{co} V, x_{1} \geq 0\right\}=\operatorname{cav} u(0)
$$

Indeed, $u$ is upper-semi-continuous, non-increasing and the hypograph of $u$ is the comprehensive set $V^{*}=V-\mathbb{R}_{+}^{2}$ associated to $V$. This implies that cav $u$ is also non-increasing, l.s.c. and its hypograph is co $V^{*}$. 


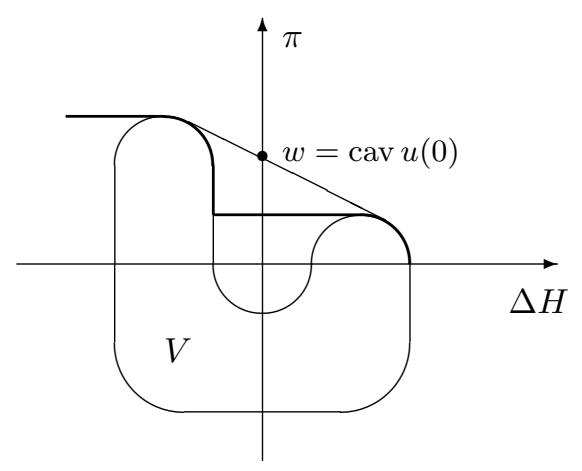

Figure 1

The bold curve is the graph of $u$.

\subsection{The main result.}

Theorem 7. The uniform minmax $v_{\infty}$ exists, $\lim _{\lambda} v_{\lambda}=\lim _{n} v_{n}=v_{\infty}$, and this value is:

$$
v_{n}=v_{\infty}=w
$$

Further, the team guarantees $w$ by an autonomous strategy.

4.3. Example. We take back the last example of section 2 i.e. the following 3player game where player 1 chooses rows, player 2 chooses columns and player 3 chooses matrices.

$$
\begin{gathered}
a \\
b
\end{gathered} \underset{\left.\begin{array}{cc}
a & b \\
0 & 0
\end{array}\right)}{\left(\begin{array}{ll}
a & b \\
0 & 0
\end{array}\right)} \begin{gathered}
\left.\begin{array}{ll}
0 & 0 \\
0 & 1
\end{array}\right) \\
R
\end{gathered}
$$

The signals are given by the moves of player 2 i.e.:

$$
\begin{aligned}
& a \\
& b
\end{aligned}\left(\begin{array}{cc}
s & b \\
s & s^{\prime} \\
s & s^{\prime}
\end{array}\right)
$$

Consider again the following strategy: the team plays the mixed action profile $\left(\frac{1}{2}, \frac{1}{2}\right) \otimes\left(\frac{1}{2}, \frac{1}{2}\right)$ at stage $2 n+1$ and at stage $2 n+2$, the team plays $(a, a)$ if $\mathbf{a}_{2 n+1}^{1}=a$ and $(b, b)$ if $\mathbf{a}_{2 n+1}^{1}=a$. This strategy consists in playing alternatively two correlation systems. Let $c_{+1}$ be the Dirac measure on $\left(\frac{1}{2}, \frac{1}{2}\right) \otimes\left(\frac{1}{2}, \frac{1}{2}\right)$ and $c_{-1}$ which puts equal weights on $(1,0) \otimes(1,0)$ and on $(0,1) \otimes(0,1)$ i.e. $c_{-1} \in \Delta(X)$ and $c_{-1}(\{(1,0) \otimes(1,0)\})=c_{-1}(\{(0,1) \otimes(0,1)\})=\frac{1}{2}$. We have $\pi\left(c_{+1}\right)=\frac{1}{4}$, $\Delta H\left(c_{+1}\right)=+1, \pi\left(c_{-1}\right)=\frac{1}{2}$ and $\Delta H\left(c_{-1}\right)=-1$ since the move of player 2 at an even stage reveals the action of player 1 at the previous stage. The so-defined strategy, playing $c_{+1}$ at odd stages and $c_{-1}$ at even stages gives an average payoff of $\frac{3}{8}$ and an average entropy variation of 0 .

We now prove the existence of strategies for players 1 and 2 that guarantee more than $\frac{3}{8}$. We just show the existence of a convex combination of two correlation systems yielding an average payoff larger than $\frac{3}{8}$ and a non-negative average entropy variation, and apply theorem 7 .

Define the correlation system $c_{\epsilon}$ which puts equal weights on $(1-\varepsilon, \varepsilon) \otimes(1,0)$ and $(\varepsilon, 1-\varepsilon) \otimes(0,1): \quad c_{\epsilon}(\{(1-\varepsilon, \varepsilon) \otimes(1,0)\})=c_{\epsilon}(\{(\varepsilon, 1-\varepsilon) \otimes(0,1)\})=\frac{1}{2}$. We have $\pi\left(c_{\varepsilon}\right)=\frac{1-\varepsilon}{2}$ and $\Delta H\left(c_{\varepsilon}\right)=h(\varepsilon)-1$ where for $\left.x \in\right] 0,1[, h(x)=$ 
$-x \log (x)-(1-x) \log (1-x), h(0)=h(1)=0$. Using that $h^{\prime}(0)=+\infty$, we deduce the existence of $\varepsilon>0$ such that $\left(\Delta H\left(c_{\varepsilon}\right), \pi\left(c_{\varepsilon}\right)\right)$ lies above the line

$$
\left\{\lambda\left(-1, \frac{1}{2}\right)+(1-\lambda)\left(1, \frac{1}{4}\right), \lambda \in[0,1]\right\}
$$

For this $\varepsilon$, there exists $0 \leq \lambda \leq 1$ such that $\lambda \Delta H\left(c_{\varepsilon}\right)+(1-\lambda) \Delta H\left(c_{+1}\right)=0$ and $\lambda \pi\left(c_{\varepsilon}\right)+(1-\lambda) \pi\left(c_{+1}\right)>\frac{3}{8}$, which implies that the team can guarantee more than $\frac{3}{8}$.

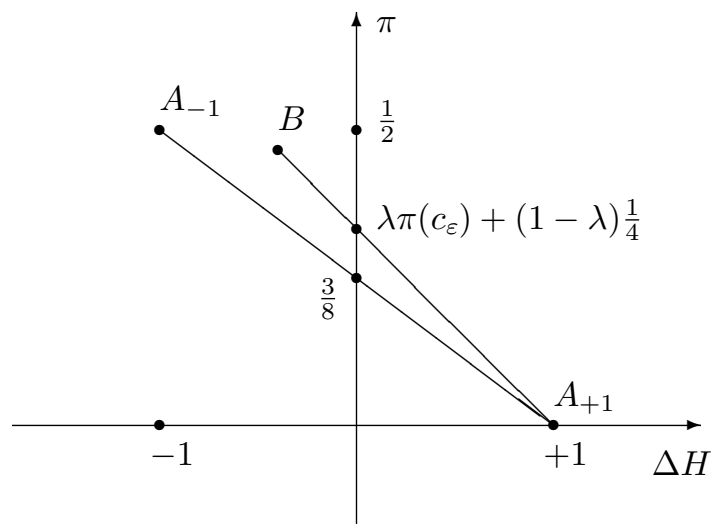

Figure 2

$$
A_{+1}=\left(\Delta H\left(c_{+1}\right), \pi\left(c_{+1}\right)\right), A_{-1}=\left(\Delta H\left(c_{-1}\right), \pi\left(c_{-1}\right)\right), B=\left(\Delta H\left(c_{\varepsilon}\right), \pi\left(c_{\varepsilon}\right)\right)
$$

\subsection{Proof of the main theorem.}

4.4.1. Player II defends $w$. We prove here that for every strategy of the team, if player $I I$ plays stage-best replies, this generates an average vector of (payoffs, entropy variation) that belongs to $V$. This will imply that no strategy for the team can guarantee a better payoff than $w$. The proof follows the same lines as for instance the papers [NO99],[NO00], [GV02].

Lemma 8. For each integer $n$ and strategy profile for the team $\sigma$ :

$$
\gamma_{n}\left(\sigma, \tau_{\sigma}\right) \leq w
$$

Proof. Let $\sigma$ be a strategy for the team and set $\tau=\tau_{\sigma}$. Let $\mathbf{a}_{m}, \mathbf{b}_{m}, \mathbf{s}_{m}$ be the sequences of random action profiles and signals associated to $(\sigma, \tau), \mathbf{h}_{m}^{I I}=$ $\left(\mathbf{b}_{1}, \mathbf{s}_{1}, \ldots, \mathbf{b}_{m-1}, \mathbf{s}_{m-1}\right)$ be the history of player $I I$ before stage $m$ and $\mathbf{h}_{m}=$ $\left(\mathbf{a}_{1}, \mathbf{b}_{1}, \mathbf{s}_{1}, \ldots, \mathbf{a}_{m-1}, \mathbf{b}_{m-1}, \mathbf{s}_{m-1}\right)$ be the total history. Let $c_{m}\left(\mathbf{h}_{m}^{I I}\right)$ be the distribution of $\sigma\left(\mathbf{h}_{m}\right)$ conditional on $\mathbf{h}_{m}^{I I}$ i.e. $c_{m}\left(\mathbf{h}_{m}^{I I}\right)$ is the correlation system at stage $m$ after history $\mathbf{h}_{m}^{I I}$ and let also $\mathbf{x}_{m}=\sigma\left(\mathbf{h}_{m}\right)$. The payoff at stage $m$ after $\mathbf{h}_{m}^{I I}$ is $\min _{b} g\left(\mathbf{E}_{\sigma, \tau}\left[\mathbf{x}_{m} \mid \mathbf{h}_{m}^{I I}\right], b\right)=\pi\left(\mathbf{c}_{m}\right)$ from the definition of $\tau$ and thus $\gamma_{n}(\sigma, \tau)=\mathbf{E}_{\sigma, \tau}\left[\frac{1}{n} \sum_{m=1}^{n} \pi\left(\mathbf{c}_{m}\right)\right]$.

We set $H_{m}=H\left(\mathbf{a}_{1}, \ldots, \mathbf{a}_{m} \mid \mathbf{h}_{m+1}^{I I}\right)$ and using the additivity of entropy, we have:

$$
\begin{aligned}
H\left(\mathbf{a}_{1}, \ldots, \mathbf{a}_{m}, \mathbf{b}_{m}, \mathbf{s}_{m} \mid \mathbf{h}_{m}^{I I}\right) & =H\left(\mathbf{b}_{m}, \mathbf{s}_{m} \mid \mathbf{h}_{m}^{I I}\right)+H_{m} \\
& =H_{m-1}+H\left(\mathbf{a}_{m}, \mathbf{b}_{m}, \mathbf{s}_{m} \mid \mathbf{h}_{m}\right)
\end{aligned}
$$


Thus,

$$
\begin{aligned}
H_{m}-H_{m-1} & =H\left(\mathbf{a}_{m}, \mathbf{b}_{m}, \mathbf{s}_{m} \mid \mathbf{h}_{m}\right)-H\left(\mathbf{b}_{m}, \mathbf{s}_{m} \mid \mathbf{h}_{m}^{I I}\right) \\
& =H\left(\mathbf{a}_{m}, \mathbf{s}_{m} \mid \mathbf{h}_{m}\right)-H\left(\mathbf{s}_{m} \mid \mathbf{h}_{m}^{I I}\right)+H\left(\mathbf{b}_{m} \mid \mathbf{h}_{m}\right)-H\left(\mathbf{b}_{m} \mid \mathbf{h}_{m}^{I I}\right) \\
& =H\left(\mathbf{a}_{m}, \mathbf{s}_{m} \mid \mathbf{h}_{m}\right)-H\left(\mathbf{s}_{m} \mid \mathbf{h}_{m}^{I I}\right) \\
& =H\left(\mathbf{a}_{m}, \mathbf{s}_{m} \mid \mathbf{x}_{m}, \mathbf{h}_{m}^{I I}\right)-H\left(\mathbf{s}_{m} \mid \mathbf{h}_{m}^{I I}\right) \\
& =\mathbf{E}_{\sigma, \tau} \Delta H\left(c_{m}\left(\mathbf{h}_{m}^{I I}\right)\right)
\end{aligned}
$$

where the second equality holds since $\mathbf{a}_{m}$ and $\mathbf{b}_{m}$ are independent conditional on $\mathbf{h}_{m}^{I I}$, the third uses that $\mathbf{b}_{m}$ is $\mathbf{h}_{m}^{I I}$-measurable and the fourth that $\left(\mathbf{a}_{m}, \mathbf{s}_{m}\right)$ depends on $\mathbf{h}_{m}$ only through $\mathbf{x}_{m}$. We deduce:

$$
\sum_{m=1}^{n} \mathbf{E}_{\sigma, \tau} \Delta H\left(c_{m}\left(\mathbf{h}_{m}^{I I}\right)\right)=H\left(\mathbf{a}_{1}, \ldots, \mathbf{a}_{n} \mid \mathbf{b}_{1}, \mathbf{s}_{1} \ldots, \mathbf{b}_{n}, \mathbf{s}_{n}\right) \geq 0 .
$$

Therefore the vector $\left(\frac{1}{n} \sum_{m=1}^{n} \mathbf{E}_{\sigma, \tau} \Delta H\left(c_{m}\left(\mathbf{h}_{m}^{I I}\right)\right), \gamma_{n}(\sigma, \tau)\right)$ is in $\operatorname{co} V \cap\left\{x_{1} \geq 0\right\}$.

4.4.2. The team guarantees $w$. We call a distribution $\mathbf{P}$ on $(A \times S)^{\infty}$ an $X$ distribution if at each stage $n$, after $\mathbf{P}$-almost every history $h=\left(a_{1}, s_{1}, \ldots, a_{n}, s_{n}\right) \in$ $H_{n}^{I}$, the distribution of $\mathbf{a}_{n+1}$ conditional on $h, \mathbf{P}\left(\mathbf{a}_{n+1} \mid h\right)$ belongs to $X$. Every autonomous strategy profile induces an $X$-distribution and conversely an $X$ distribution defines an autonomous strategy profile.

Given an autonomous strategy profile $\sigma$ or equivalently an $X$-distribution, consider the random correlation system at stage $n$ : given $\mathbf{h}_{n}^{I I}, \mathbf{c}_{n}$ is the distribution of $\sigma\left(h_{n}^{I}\right)$ given $\mathbf{h}_{n}^{I I}$. The random variable $\mathbf{c}_{n}$ is $\mathbf{h}_{n}^{I I}$-measurable with values in $C=\Delta(X)$. Define the empirical distribution of correlation systems up to stage $n$ as the random variable:

$$
\mathbf{d}_{n}=\frac{1}{n} \sum_{m \leq n} \epsilon_{\mathbf{c}_{m}}
$$

where $\epsilon_{c}$ denotes the Dirac measure on $c$. The random variable $\mathbf{d}_{n}$ has values in $D=\Delta(C)$. If we let $\delta=\mathbf{E}_{\sigma} \mathbf{d}_{n}$ be the element of $D$ such that for any real-valued continuous function $f$ on $C, \mathbf{E}_{\sigma}\left[\int f(b) d \mathbf{d}_{n}(b)\right]=\int f(b) d \delta(b)$, we express the average payoff as follows:

$$
\gamma_{n}\left(\sigma, \tau_{\sigma}\right)=\mathbf{E}_{\delta} \pi
$$

We use the following result from Gossner and Tomala [GT04]:

Theorem 9 ([GT04], thm 9.). For every $\delta \in \Delta(C)$ such that $\mathbf{E}_{\delta} \Delta H \geq 0$, there exists an $X$-distribution $\mathbf{P}$ on $(A \times S)^{\infty}$ such that $\mathbf{E}_{\mathbf{P}} \mathbf{d}_{n}$ weak-* converges to $\delta$.

Since any $X$-distribution $\mathbf{P}$ corresponds to an autonomous strategy, we get:

Lemma 10. The team guarantees $\sup \left\{\mathbf{E}_{\delta} \pi \mid \delta \in \Delta(C), \mathbf{E}_{\delta} \Delta H \geq 0\right\}$.

Proof. For each $\delta$ such that $\mathbf{E}_{\delta} \Delta H \geq 0$, the previous theorem yields the existence of an autonomous strategy $\sigma$ such that $\lim _{n} \gamma_{n}\left(\sigma, \tau_{\sigma}\right)=\mathbf{E}_{\delta} \pi$. Lemma 2 implies that this strategy guarantees $\mathbf{E}_{\delta} \pi$.

We conclude then by the following lemma:

Lemma 11. $\sup \left\{\mathbf{E}_{\delta} \pi \mid \delta \in \Delta(C), \mathbf{E}_{\delta} \Delta H \geq 0\right\}=w$.

Proof. Immediate since the set of vectors $\left(\mathbf{E}_{\delta} \Delta H, \mathbf{E}_{\delta} \pi\right)$ as $\delta$ varies in $\Delta(C)$ is co $V$. 
4.4.3. Convergence of $v_{n}$ and $v_{\lambda}$. It is clear from lemmata 8,10 and 11 that $v_{\infty}$ exists and $v_{\infty}=\lim _{n} v_{n}=w$. For the convergence of $v_{\lambda}$, note that the discounted payoff is a convex combination of the average payoffs (see Lehrer and Sorin [LS92]):

$$
\gamma_{\lambda}(\sigma)=\sum_{n \geq 1}(1-\lambda)^{2} n \lambda^{n-1} \gamma_{n}(\sigma)
$$

From lemma 8 we get $v_{\lambda} \leq w$ and the proof is completed by the following lemma.

Lemma 12. $\forall \varepsilon>0, \exists \sigma, \exists \lambda_{0}$, such that $\forall \lambda \geq \lambda_{0}, \gamma_{\lambda}\left(\sigma, \tau_{\sigma}\right) \geq w-\varepsilon$.

Proof. For $\varepsilon>0$, choose $\sigma$ autonomous such that $\gamma_{n}\left(\sigma, \tau_{\sigma}\right) \geq w-\frac{\varepsilon}{2}$. Define a cyclic strategy $\sigma^{*}$ as follows: play $\sigma$ until stage $n$ and restart this strategy every $n$ stages. Set $y_{m}$ as the expected payoff under $\left(\sigma^{*}, \tau_{\sigma^{*}}\right)$ at stage $m$. Since $\sigma^{*}$ is cyclic, $\tau_{\sigma^{*}}$ is also cyclic and:

$$
\left.\gamma_{\lambda}\left(\sigma^{*}, \tau_{\sigma^{*}}\right)=\sum_{m=1}^{n}(1-\lambda) \lambda^{m-1} y_{m}+\lambda^{n} \gamma_{\lambda}\left(\sigma^{*}, \tau_{\sigma^{*}}\right)\right)
$$

So,

$$
\gamma_{\lambda}\left(\sigma^{*}, \tau_{\sigma^{*}}\right)=\sum_{m=1}^{n}(1-\lambda) \frac{\lambda^{m-1}}{1-\lambda^{n}} y_{m}
$$

Then, $\lim _{\lambda \rightarrow 1} \gamma_{\lambda}\left(\sigma^{*}, \tau_{\sigma^{*}}\right)=\frac{1}{n} \sum_{m=1}^{n} y_{m} \geq w-\frac{\varepsilon}{2}$ which ends the proof of the lemma.

\section{Perfect and trivial observation}

5.1. Perfect observation. We say that the observation is perfect when the signal $s$ reveals the action profile $a$ i.e. $a \neq a^{\prime} \Rightarrow \operatorname{supp} q(\cdot \mid a) \cap \operatorname{supp} q\left(\cdot \mid a^{\prime}\right)=\emptyset$. It is well known that in this case, the max min of the repeated game is the independent max min of player $I I, v_{\infty}=\max _{x \in X} \min _{b} g(x, b)$. We verify now that our main theorem gives the same value.

Since the observation is perfect, $H(\mathbf{a} \mid \mathbf{s})=0$ and $\Delta H(c)=H(\mathbf{s} \mid \mathbf{x})-H(\mathbf{s}) \leq 0$ for each correlation system $c$ and $\Delta H(c)=0$ if and only if $\mathbf{s}$ (and thus a) in independent of $\mathbf{x}$. This implies that $\Delta H(c)=0$ if and only if $c$ is a Dirac measure on some $x \in X$. We let $C_{d}$ be the set of correlation systems whose support is a subset of $\left\{\epsilon_{x}, x \in X\right\}$ where $\epsilon_{x}$ denotes the Dirac measure on $x$. From the above discussion it follows that for every distribution $\delta, \mathbf{E}_{\delta} \Delta H \geq 0$ if and only if the support of $\delta$ is a subset of $C_{d}$. This has a clear interpretation: if the observation is perfect, at each stage, the next moves of the team are independent conditional on the signals of player $I I$. Thus, $w=\sup \left\{\pi\left(\epsilon_{x}\right), x \in X\right\}$ that is $w=\max _{x \in X} \min _{b} g(x, b)$.

5.2. Trivial observation. We say that the observation is trivial when the signal $s$ does not depend on the action profile $a$. In this case, it is clear that by some exchange of messages, the team can perfectly correlate their action profile and thus $v_{\infty}=\max _{x \in \Delta(A)} \min _{b} g(x, b)$ which is the correlated minmax of player $I I$. Applying our main theorem, we remark that if observation is trivial $\Delta H(c) \geq 0$ for each $c$ and thus every distribution $\delta$ verifies $\mathbf{E}_{\delta} \Delta H \geq 0$, therefore $w=\sup \{\pi(c), c \in C\}=\max _{x \in \Delta(A)} \min _{b} g(x, b)$.

\section{Numerical EXAMPLES}

The $\max \min w$ is characterized as cav $u(0)$ with $u(h)=\max \{\pi(c) \mid c \in$ $C, \Delta H(c) \geq h\}$ so the numerical computation of $w$ constits in computing the function $u(h)$. Note that for each $h \in \mathbb{R}$, either $\operatorname{cav} u(h)=u(h)$ or cav $u$ is linear on some interval containing $h$. Thus, either cav $u(0)=\pi(c)$ for some $c$ s.t. $\Delta H(c) \geq 0$ or there exists $c_{1}, c_{2}$ and $\lambda \in(0,1)$ s.t. $\operatorname{cav} u(0)=\lambda \pi\left(c_{1}\right)+(1-\lambda) \pi\left(c_{2}\right)$ and $\lambda \Delta H\left(c_{1}\right)+(1-\lambda) \Delta H\left(c_{2}\right) \geq 0$. In the first case, the optimal strategy can be 
regarded as "stationary" (in the space of correlation systems) since only one correlation system is used at almost all stages. In the second case, the strategy has two phases. Assume $\Delta H\left(c_{1}\right)>0$, in a first phase the optimal strategy plays $c_{1}$ to accumulate entropy and in a second phase, the optimal strategy plays $c_{2}$ that spends entropy and yields a good payoff. The relative lengths of these phases are $(\lambda, 1-\lambda)$. We give now examples illustrating both cases. The computation of $u(h)$ is generally difficult and has lead to two articles: Gossner et al. [GLT03] and Goldberg [Gol03].

6.1. An example of optimal "stationary" correlation. The paper by Gossner et al. [GLT03] is devoted to the computation of the max min for the game given last example of section 2 :

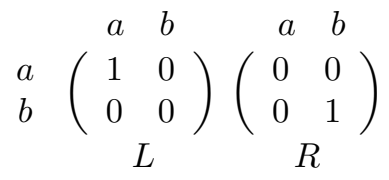

where the signal is given by the matrix:

$$
\begin{aligned}
& a \\
& b
\end{aligned} \quad\left(\begin{array}{cc}
a & b \\
s & s^{\prime} \\
s & s^{\prime}
\end{array}\right)
$$

In this case, Gossner et al. [GLT03] prove that there exists a correlation system $c$ such that $\Delta H(c)=0$ and $w=\pi(c)$. This system can be written:

$$
c=\frac{1}{2} \epsilon_{(x, 1-x) \otimes(x, 1-x)}+\frac{1}{2} \epsilon_{(1-x, x) \otimes(1-x, x)}
$$

where $0<x<\frac{1}{2}$ is such that $\Delta H(c)=2 H(x, 1-x)-1=0$. Numerically, this gives $w \cong 0.401$.

6.2. A example of optimal convexification. Another example is studied by Goldberg [Gol03]. Consider the game where payoffs for players 1 and 2 are given by:

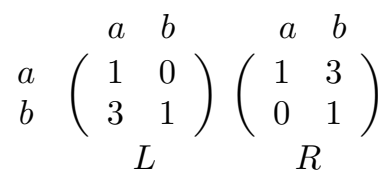

Players 1 and 2 observe the action profiled played, and player 3 observes a signal on player 1 and 2's action given by the matrix:

$$
\begin{aligned}
& a \\
& b
\end{aligned}\left(\begin{array}{cc}
a & b \\
s & s^{\prime \prime} \\
s^{\prime} & s
\end{array}\right)
$$

The independent max min is $\frac{5}{4}$ and is obtained by the mixed action $\left(\frac{1}{2}, \frac{1}{2}\right) \otimes\left(\frac{1}{2}, \frac{1}{2}\right)$. The correlated maxmin is $\frac{3}{2}$ and is obtained by the distribution of actions $\frac{1}{2}(1,0) \otimes(1,0)+\frac{1}{2}(0,1) \otimes(0,1)$.

Using theorem 7, Goldberg [Gol03] proves that the minmax is $\frac{4}{3}$ and is achieved by the following strategy: play $\left(\frac{1}{2}, \frac{1}{2}\right) \otimes\left(\frac{1}{2}, \frac{1}{2}\right)$ until the signal $s$ appears. If $s$ appears at stage $n$, play $(a, b)$ at stage $n+1$ if $(a, a)$ was played at stage $n$, and play $(b, a)$ if $(b, b)$ was played. Resume playing $\left(\frac{1}{2}, \frac{1}{2}\right) \otimes\left(\frac{1}{2}, \frac{1}{2}\right)$ at stage $n+2$.

Under this strategy, two correlations systems are used: $c_{1}$ which is the Dirac measure on $\left(\frac{1}{2}, \frac{1}{2}\right) \otimes\left(\frac{1}{2}, \frac{1}{2}\right)$ and $c_{2}=\frac{1}{2} \epsilon_{(0,1) \otimes(1,0)}+\frac{1}{2} \epsilon_{(1,0) \otimes(0,1)}$. The strategy induces an irreducible Markov chain on the finite state space $\left\{c_{1}, c_{2}\right\}$ and in the long run it spends two thirds of the time in $c_{1}$. Since $\pi\left(c_{1}\right)=\frac{5}{4}$ and $\pi\left(c_{2}\right)=\frac{4}{3}$ this 
strategy yields an average payoff of $\frac{4}{3}$. We check now that the average entropy variation is non-negative.

Under $c_{1}$, with probability $\frac{1}{2}$, players 1 and 2 play different actions and thus the action profile is revealed by the signal: no entropy is gained. With probability $\frac{1}{2}$ players 1 and 2 play the same action inducing the signal $s$. The conditional distribution puts then equal probabilities on $(a, a)$ and $(b, b): 1$ bit of entropy is gained. Thus $\Delta H\left(c_{1}\right)=\frac{1}{2} \times 0+\frac{1}{2} \times 1=\frac{1}{2}$. Under $c_{2}$, players 1 and 2 play different actions with probability 1 and their action profile is revealed by the signal. All entropy is lost and $\Delta H\left(c_{1}\right)=-1$. One has $\frac{2}{3} \Delta H\left(c_{1}\right)+\frac{1}{3} \Delta H\left(c_{2}\right)=0$.

\section{A FolK Theorem}

The previous examples show 3 player games in which our main theorem allows to characterize the individually rational payoff of one player in the repeated game. We now present a signalling structure for which our theorem allows for a characterization of all individually rational payoffs.

Consider a game in which player the set of players is $I=\{1, \ldots, n\}, n \geq 4$, and $i$ 's finite action set is $A^{i}$. Players $i, 1<i<n$ have perfect observation: they observe $s^{i}=\left(a^{1}, \ldots, a^{n}\right)$. Player 1 observes every player but player $n$ : his signal is $s^{1}=\left(a^{1}, \ldots, a^{n-1}\right)$. Player $n$ observes every player but player 1 : his signal is $s^{n}=\left(a^{2}, \ldots, a^{n}\right)$. This structure of signals is represented in Renault and Tomala [RT98] by a graph whose nodes are the players and where there is an edge between $i$ and $j$ whenever $i$ and $j$ monitor each other. The graph described here is 2-connected: there are at least two distinct paths from $i$ to $j$ for each pair $(i, j)$.

Since each player $1<i<n$ has perfect observation, his individually rational levels $v^{i}$ in the repeated game equals his minmax in mixed strategies of the one-shot game.

Consider a modified version of the repeated game in which player 1 also observes the moves of player $n$. This modified signalling structure between the team of players $\{1, \ldots, n-1\}$ and player $n$ fulfill the conditions of our theorem. Let then $v^{n}$ be the uniform min max of player $n$ in the modified repeated game. Since players $\{1, \ldots, n-1\}$ can push player $n$ 's payoff down to $v^{n}$ with autonomous strategies, they can also push player $n$ down $v^{n}$ in the original game. On the other hand, since player $n$ can defend $v^{n}$ in the modified repeated game, he can also defend $v^{n}$ in the original one. Hence the individually rational level of player $n$ in the original game is $v^{n}$. The same reasoning shows that our theorem applies to a characterization of the $\min \max v^{1}$ for player 1 .

Let $\operatorname{cog}(A)$ be the set of feasible payoffs, and $I R=\left\{x \in \mathbb{R}^{n}, x^{i} \geq v^{i}\right\}$ be the set of individually rational payoffs. Renault and Tomala [RT98] prove that when the signals are represented by a 2-connected graph, the set of uniform equilibrium payoffs is $\operatorname{co} g(A) \cap I R$.

Lehrer [Leh90] characterizes Nash equilibrium payoffs for all repeated games having a semi-standard signalling structure. Our example constitutes -as far as we know- the only other $n$-player signalling structure for which a characterization of Nash equilibrium payoffs is known for all payoff functions.

\section{EXTENSIONS}

8.1. More general signals. Theorem 7 is still valid if team players do not fully observe the move $b$ of player $I I$, i.e. if whenever player $I I$ plays $b$, a signal $r$ is drawn according to some distribution $p(\cdot \mid b)$ that depends on $b$ only and publicly announced. The quantity $w$ can still be guaranteed with autonomous strategies and on another hand the proof of lemma 8 extends perfectly. 


\section{REFERENCES}

[Aum74] R.J. Aumann. Subjectivity and correlation in randomized strategies. Journal of Mathematical Economics, 1:67-95, 1974.

[CT91] T. M. Cover and J. A. Thomas. Elements of information theory. Wiley Series in Telecomunications. Wiley, 1991.

[For86] F. Forges. An Approach to Communication Equilibria. Econometrica, 54:1375-1385, 1986.

[GLT03] O. Gossner, R. Laraki, and T. Tomala. On the optimal use of coordination. mimeo, 2003.

[Gol03] Y. Goldberg. On the minmax of repeated games with imperfect monitoring: a computational example. mimeo, 2003.

[GT04] O. Gossner and T. Tomala. Empirical distributions of Beliefs under imperfect observation. mimeo, Cahier du Ceremade, 2004.

[GV02] O. Gossner and N. Vieille. How to play with a biased coin? Games and Economic Behavior, 41:206-226, 2002.

[Leh90] E. Lehrer. Nash equilibria of $n$ player repeated games with semi-standard information. International Journal of Game Theory, 19:191-217, 1990.

[LS92] E. Lehrer and S. Sorin. A uniform tauberian theorem in dynamic programming. Mathematics of Operations Research, 17:303-307, 1992.

[MSZ94] J.-F. Mertens, S. Sorin, and S Zamir. Repeated games. CORE discussion paper 9420$9422,1994$.

[NO99] A. Neyman and D. Okada. Strategic entropy and complexity in repeated games. Games and Economic Behavior, 29:191-223, 1999.

[NO00] A. Neyman and D. Okada. Repeated games with bounded entropy. Games and Economic Behavior, 30:228-247, 2000.

[RT98] J. Renault and T. Tomala. Repeated proximity games. International Journal of Game Theory, 27:539-559, 1998.

[RT00] J. Renault and T. Tomala. Communication equilibrium payoffs of repeated games with imperfect monitoring. Cahiers du CEREMADE 0034, Université Paris Dauphine, Paris, 2000.

[vSK97] B. von Stengel and D. Koller. Team max min equilibria. Games and Economics Behavior, 21:309-321, 1997.

CERAS, URA CNRS 2036

E-mail address: 0livier.Gossner@mail.enpc.fr

CEREMADE, UMR CNRS 7534 Université PARIS 9 - DAuphine

E-mail address: tomala@ceremade.dauphine.fr 